\title{
Mejorando la percepción sobre la inteligencia: una intervención breve para alumnos de Educación Secundaria
}

\author{
Elena Medina-Garrido ${ }^{1}$ y Jaime León ${ }^{2}$ \\ ${ }^{1}$ Consejería de Educación, Gobierno de Canarias, Las Palmas \\ ${ }^{2}$ Departamento de Educación, Universidad de Las Palmas de Gran Canaria, Las \\ Palmas
}

\section{España}

Correspondencia: Jaime León. Departamento de Educación. Universidad de Las Palmas de Gran Canaria. C/Santa Juana de Arco, 1. 35004-Las Palmas. España. E-mail: jaime.leon@ulpgc.es

(C) Education \& Psychology I+D+i and Ilustre Colegio Oficial de la Psicología de Andalucía Oriental (Spain) 


\section{Resumen}

Introducción. En Educación Secundaria es frecuente encontrarse con alumnos que piensan que tal vez no sean lo suficientemente inteligentes como para superar con éxito esta etapa educativa. Pensar en la inteligencia como algo fijo o modificable influye en el rendimiento académico, por lo que nos preguntamos si una intervención breve puede cambiar las ideas del alumnado sobre su inteligencia. El objetivo principal de esta investigación fue diseñar y analizar la eficacia de una intervención, para que el alumnado piense que la inteligencia no es algo innato e inmodificable, sino que todos tenemos un potencial intelectual y que depende de nosotros mismos trabajar para desarrollarlo.

Método. El programa se diseñó siguiendo los principios de las intervenciones breves, y se llevó a cabo con alumnos de $1^{\circ}$ de Educación Secundaria Obligatoria. Para analizar su eficacia se realizó un estudio con grupo cuasi-experimental y de cuasi-control, con una evaluación previa y dos posteriores a la intervención.

Resultados. Mediante un modelo multinivel se observó que la trayectoria del grupo experimental fue diferente a la del grupo control, obteniendo evidencias de que los alumnos del grupo experimental disminuyeron sus creencias acerca de la inteligencia como algo estable e inmodificable.

Discusión y conclusiones. Este trabajo puede servir como punto de partida para que futuros trabajos mejoren el rendimiento académico de una forma sencilla, replicable y fácilmente escalable.

Palabras Clave: ideas implícitas sobre la inteligencia; desmotivación; intervención; estudio experimental; educación secundaria. 


\begin{abstract}
Introduction. Holding a fix or an incremental mindset influence academic performance; we wonder if an intervention would change students' mindsets. The main goal of this study was to design and analyse the effectiveness of an easy to scale intervention to diminish students' belief about intelligence as something innate and fix, and think that we all have an intellectual potential that depends on ourselves to work it and, thus, maximize our performance.
\end{abstract}

Method. The program was designed following the guidelines of brief interventions, because of their numerous benefits, and it was tested with students from first year of Compulsory Secondary Education. To test its efficacy, we ran a study comparing an experimental and control group with one prior and two post-intervention assessments.

Results. We observed using a multilevel model that the trajectory of the experimental group was different from the control group, obtaining evidence that students in the experimental group decreased their beliefs about intelligence as something fix.

Discussion and Conclusion. This work may serve as a starting point for future work to improve academic performance in a simple, easily replicable and scalable way.

Keywords: mindsets, motivation, intervention, experimental design, secondary education. 


\section{Introducción}

En Educación Secundaria es frecuente encontrarse con alumnos que piensan que tal vez no sean lo suficientemente listos como para superar con éxito esta etapa educativa (Paunesku et al., 2015; Yeager et al., 2016). La motivación y la implicación de los alumnos disminuye al pasar de primaria a secundaria (Davidson, Gest y Welsh, 2010; Roeser y Eccles, 1998), y son varias las razones que pueden explicar este hecho: hay más alumnos en las clases, los estudiantes tienen muchos profesores distintos, por lo que la relación con ellos es más impersonal y, además, los contenidos de las clases son más difíciles (Goodenow, 1993). Todo esto lleva a algunos alumnos a pensar que no son lo suficientemente inteligentes como para alcanzar los objetivos que se les exige (Anderman, 2003; Wentzel, 2009).

Este tema ha sido estudiado por varios autores, entre ellos Dweck (2006), quien, desde su teoría de las ideas implícitas de la inteligencia o mindsets, plantea que existen alumnos que piensan que su inteligencia es algo fijo, mientras que otros alumnos piensan que es algo que se puede modificar. Esta autora con otros expertos (Blackwell, Trzesniewski y Dweck, 2007; Romero, Master, Paunesku, Dweck y Gross, 2014), ha analizado cómo la percepción o idea que tienen los alumnos de su propia inteligencia está relacionada con el rendimiento académico: los alumnos que piensan en la inteligencia como algo modificable obtienen mejores resultados. Además, se han realizado intervenciones en EEUU basadas en la teoría de Dweck (1999) que han demostrado que se puede cambiar la percepción que los alumnos tienen sobre su capacidad intelectual (Blackwell et al., 2007; Donohoe, Topping y Hannah, 2012).

Habitualmente estas intervenciones se han desarrollado en un período corto de tiempo bajo el paradigma de las intervenciones breves (Walton y Cohen, 2011; Yeager et al., 2014) que presentan ventajas como: la facilidad de aplicación, el enfoque específico de un determinado problema, la discreción y la atribución de la mejora a causas internas del propio alumno (Yeager y Walton, 2011). Incluso intervenciones de una sola sesión han demostrado su eficacia para, por ejemplo, mejorar el estilo parental (Lim, Tormshak y Dishion, 2005), aumentar la concienciación sobre los riesgos de las enfermedades de transmisión sexual (Thurstone, Riggs, Klein y Mikulich-Gilbertson, 2007) o disminuir la violencia de género (Crane y Eckhardt, 2013).

\section{Teoría de las ideas implícitas de la inteligencia}


¿En qué consiste exactamente la teoría enunciada por Dweck sobre las ideas preconcebidas de los alumnos a cerca de su inteligencia? Blackwell, Trzesniewski y Dweck (2007) explican que los estudiantes pueden tener dos visiones acerca de la naturaleza de su inteligencia: unos piensan que la inteligencia es algo estable, no modificable y que depende de la genética, y otros que no es estable, sino que se puede mejorar y que depende del aprendizaje y el esfuerzo. Sin embargo, esto no implica que estos últimos piensen que todo el mundo tiene el mismo potencial: lo que piensan es que cada individuo puede desarrollar su capacidad intelectual hasta llegar al máximo de su propio potencial (Hong, Chiu, Dweck, Lin y Wan, 1999; Inglés et al., 2015). Estudios correlacionales y experimentales han demostrado que estas dos formas de pensar acerca de la inteligencia promueven que los estudiantes piensen, sientan y se comporten de manera diferente (Rattan, Good y Dweck, 2012; Verniers y Martinot, 2015).

\section{Evidencias correlacionales}

Dweck y Leggett (1988) observaron que pensar en la inteligencia de una u otra forma se relaciona con la motivación y la autorregulación; en concreto, concluyeron que quienes creen que la capacidad intelectual se puede desarrollar tratan de realizar tareas que promueven la adquisición de la habilidad intelectual, y se esfuerzan para superar las dificultades, mientras que quienes piensan en la inteligencia como algo estable, ante una dificultad tienden a retirarse y a no esforzarse.

También se ha observado que estas dos formas de pensar se relacionan con el aprendizaje y el rendimiento académico. Chen y Pajares (2010) realizaron un estudio en EEUU con una muestra de 508 individuos que estaban en su primer año de Educación Secundaria que reveló que la visión modificable acerca de la inteligencia predecía de forma indirecta las calificaciones a través de una serie de variables motivacionales y autorreguladas. En la misma línea, Luo, Lee, Ng y Wei Ong (2014), en un estudio con 273 alumnos de Singapur (correspondiente a segundo de Educación Secundaria del sistema educativo español), obtuvieron evidencias de que pensar en la inteligencia hacia las Matemáticas como algo que se puede mejorar está relacionado directamente con el hecho de ver las clases de Matemáticas como algo divertido y con sentirse satisfecho al creer que se les da bien la materia, e inversamente relacionado con el aburrimiento y la preocupación por fracasar en clase. Además, estos autores observaron que la creencia de la inteligencia matemática como algo mejorable predecía el rendimiento en la asignatura de Matemáticas en dos sentidos: 
positivamente, a través de la satisfacción, y negativamente, mediante la preocupación por fracasar.

\section{Intervenciones previas}

Blackwell et al. (2007) realizaron una intervención con una muestra de 91 alumnos que se dividió en un grupo experimental y un grupo control. Los alumnos tenían entre 12 y 14 años y se caracterizaban por tener bajo rendimiento en Matemáticas. La intervención consistió en ocho sesiones de 25 minutos en las que se enseñó a los estudiantes que la inteligencia es mejorable y que puede desarrollarse; en concreto, en las dos primeras sesiones se enseñó la anatomía del cerebro y el funcionamiento de las neuronas y neurotransmisores. En la tercera y cuarta sesión, se leyó un texto titulado "cómo aumentar tu inteligencia" y a continuación se realizó una actividad sobre el texto. En las dos siguientes sesiones se trabajó sobre los estereotipos en cuanto a género y raza, y también se trabajaron las técnicas de estudio. Finalmente, en las dos últimas sesiones se generó un debate en clase sobre cuatro aspectos: 1) el cerebro se hace más fuerte cuanto más se ejercita; 2) a medida que aprendes te haces más inteligente; 3) ser inteligente depende de uno mismo; y 4) utilizar etiquetas como "el empollón" o "el tonto" promueve que los alumnos no se atrevan a esforzarse por miedo a ser "el tonto" o "el raro" de la clase. Se debe tener en cuenta que las sesiones uno, dos, cinco y seis se llevaron a cabo tanto en el grupo experimental como en el grupo control. Se observó que inmediatamente antes de la intervención las notas en Matemáticas habían bajado con respecto a la primavera del curso anterior; sin embargo, tras la intervención las notas del grupo experimental dejaron de disminuir, mientras que las del grupo control continuaron disminuyendo.

Donohoe et al. (2012) realizaron una intervención con una muestra de 33 estudiantes escoceses de entre 13 y 14 años, 18 en el grupo experimental y 15 en el de control. La autora principal del estudio era profesora del grupo. La intervención se realizó a través de un programa informático donde los alumnos, acompañados de tres personajes interactivos, tenían que realizar una serie de actividades y retos. El programa se estructuró en cuatro sesiones de 40 minutos: la primera, sobre los aspectos básicos del cerebro; la segunda, sobre el comportamiento del cerebro; la siguiente, sobre el desarrollo del cerebro y la última, sobre estimulantes del cerebro. Observaron que el grupo control no modificó su percepción sobre la inteligencia, mientras que el grupo experimental sí lo hizo, pero pasados tres meses de la intervención su concepción sobre la inteligencia pasó a ser similar a la inicial. 


\section{Las intervenciones breves}

Como ya se ha señalado, en el contexto educativo las intervenciones breves tienen una serie de ventajas como: a) ser de fácil aplicación, b) estar dirigidas únicamente al problema a mejorar, c) ser más discretas, porque el alumnado no tiene que ir a clases extraordinarias o tener un tutor de apoyo, por lo que no sentirá el estigma de "ser un mal alumno" y d) conseguir que el alumno explique su mejora por razones internas en lugar de por causas externas, como las clases extraordinarias o el profesor de apoyo (Yeager, Walton y Cohen, 2013).

El objetivo de estas intervenciones no es profundizar en contenidos académicos o técnicas de estudio, sino que se trata de trabajar sobre los pensamientos, sentimientos y creencias de los alumnos (Lazowski y Hulleman, 2016; Spitzer y Aronson, 2015). Este tipo de intervenciones ha despertado la desconfianza de algunos profesionales de la educación, sin embargo, varios investigadores que han aplicado intervenciones breves han observado efectos muy positivos sobre el rendimiento académico (Aronson, Fried y Good, 2002; Cohen, Garcia, Purdie-Vaughns, Apfel y Brzustoski, 2009). Algunos ejemplos de intervenciones breves eficaces son las de Hulleman y Harackiewicz (2009) y Morisano, Hirsh, Peterson, Pihl y Shore (2010). Hulleman y Harackiewicz (2009), basándose en la teoría expectancy-value (Eccles, 1983; Wigfield y Eccles, 2000), realizaron un estudio experimental con asignación aleatoria al grupo control $(\mathrm{n}=126)$ y experimental $(\mathrm{n}=136)$. Durante un semestre todos los alumnos tenían que hacer cada tres o cuatro semanas un breve trabajo acerca de la asignatura de ciencias; la diferencia era que los del grupo experimental tenían que escribir sobre en qué podrían emplear ellos lo aprendido en clase, mientras que los del grupo control tenían que hacer un resumen de lo trabajado en clase. Los investigadores observaron una mejora de las calificaciones de los alumnos del grupo experimental frente a los del grupo control.

Morisano et al. (2010), partiendo del modelo del establecimiento de objetivos (Locke y Latham, 1990, 2002), analizaron la eficacia de una intervención en la que se buscaba simplemente que los alumnos universitarios establecieran unos objetivos claros y específicos. Para realizar la intervención los alumnos $(n=45)$ necesitaban acceder a una plataforma online donde se les daba una serie de instrucciones para que pudieran establecer unos objetivos concretos. Los investigadores observaron que, en comparación con el grupo control, los alumnos del grupo experimental obtuvieron mejores calificaciones. 
Por último, resulta interesante la intervención breve llevada a cabo por un profesor el primer día de clases para mejorar la motivación de alumnos universitarios (McGinley y Jones, 2014). El grupo experimental estuvo formado por alumnos de dos clases, 14 alumnos de primer curso y 39 alumnos de cuarto curso. La intervención comenzó dividiendo la clase en grupos y haciéndoles debatir sobre la utilidad y lo interesante de la asignatura. Además, el profesor respondió a las preguntas que hicieron los alumnos y se aprendió sus nombres. Los resultados fueron positivos, se obtuvieron evidencias sobre cómo la motivación de los estudiantes se había incrementado en cuanto al interés en la materia y en cuanto a la relación con el profesor.

\section{El presente estudio: objetivos e hipótesis}

Debido a la desmotivación por parte del alumnado de Educación Secundaria al creer que no son suficientemente inteligentes para afrontar los retos académicos de esta etapa educativa, y teniendo en cuenta la eficacia e importancia de intervenciones breves en el contexto educativo (Yeager et al., 2013; Yeager y Walton, 2011), nos preguntamos: ¿puede una intervención breve, basada en la teoría de las ideas implícitas de la inteligencia (Dweck, 1999) y en otras intervenciones realizadas en relación con esta teoría (Blackwell et al., 2007; Donohoe et al., 2012), modificar las ideas preconcebidas que tienen los alumnos sobre la inteligencia? En el caso de que sí las modifique, ¿estos cambios perdurarán por un mes? Esto nos permitirá saber si los beneficios de esta intervención se deberán buscar a largo o a corto plazo.

Con esta investigación no se busca demostrar que este tipo de intervenciones mejora el rendimiento académico, ya que esto ya lo han trabajado otros autores (Blackwell et al., 2007; Paunesku et al., 2015; Yeager et al., 2016), sino que el objetivo de nuestra investigación es diseñar una intervención que pueda ser aplicada en el aula y que cambie la percepción de la inteligencia de los alumnos produciéndose así una mejora de su motivación, o dicho de otra forma, buscamos que el alumnado piense que todos tenemos un potencial intelectual y que depende de nosotros mismos trabajar para desarrollarlo, en lugar de pensar que la inteligencia es algo innato e inmodificable. En concreto, nuestra hipótesis de investigación es que la percepción de inteligencia (mindset) tras la intervención será diferente entre el grupo que recibe la intervención (experimental) y el grupo que no la recibe (control), concretamente esperamos que el grupo experimental se caracterice por un mayor pensamiento de la inteligencia como una característica modificable. 


\section{Método}

\section{Participantes}

Los participantes del estudio fueron 47 alumnos de $1^{\circ}$ de Educación Secundaria de dos institutos de Gran Canaria (España). El grupo experimental estuvo formado por 26 participantes (13 alumnos y 13 alumnas) que pertenecían a un instituto, con una edad media de 13.13 años (desviación típica 0.53), mientras que el grupo control estuvo formado por 21 alumnos (6 alumnos y 15 alumnas) que, para evitar una posible contaminación, estudiaban en otro centro cercano con características similares y con una edad media de 13.19 años (desviación típica 0.54). Conviene aclarar que la muestra inicial era de 55 estudiantes, pero se observó que hubo alumnos diagnosticados con necesidades específicas de apoyo educativo que no comprendían los ítems del cuestionario; por esa razón, aunque estuvieron presentes durante la intervención, fueron excluidos del estudio y no se tuvieron en cuenta sus respuestas a la hora de analizar los datos.

\section{Instrumentos}

Para analizar si los estudiantes pensaban que su inteligencia era fija o modificable se utilizaron tres ítems del cuestionario de Dweck (1999) en una escala tipo Likert desde 1 (totalmente en desacuerdo) a 7 (totalmente de acuerdo). Los ítems utilizados fueron los siguientes: "Un estudiante no puede cambiar su inteligencia fácilmente", "Un estudiante posee una determinada inteligencia y no puede hacer mucho para cambiarla", "Un estudiante puede aprender cosas nuevas, pero no puede cambiar su inteligencia”. Esta escala ha sido utilizada en estudios previos con evidencias de fiabilidad y validez (Erdley y Dweck, 1993; Erdley, Loomis, Cain, Dumas-Hines y Dweck, 1997; Luo et al., 2014), tanto en su versión original como en su versión reducida de tres ítems. Para analizar la fiabilidad del cuestionario empleado, se utilizó el alfa de Cronbach. En este estudio en la primera (T1), segunda (T2) y tercera (T3) evaluación fue $\alpha_{\mathrm{t} 1}=.77, \alpha_{\mathrm{t} 2}=.75$ y $\alpha_{\mathrm{t} 3}=.84$.

\section{Procedimiento}

Para analizar la eficacia de la intervención se realizaron tres evaluaciones utilizando el cuestionario descrito. La primera, dos semanas antes de empezar la intervención, la segunda, después de la intervención y la tercera, un mes después de la segunda evaluación. El grupo experimental y el grupo control provenían de diferentes institutos que fueron elegidos porque los autores del trabajo ya los conocían. Estos dos institutos están cerca uno del otro, además, 
el perfil del alumnado y del profesorado es similar. Se decidió elegir grupos de diferentes institutos para disminuir el riesgo de contaminación, es decir, disminuir el riesgo de que los alumnos del grupo que recibe la intervención le cuenten al grupo que no recibe la intervención qué trabajaron.

La intervención se llevó a cabo en una sesión de tutoría de 55 minutos y se dividió en cuatro partes. El objetivo específico de la primera parte fue explicar que el cerebro es como un músculo y como tal, puede ser ejercitado para mejorar el rendimiento. Después de la explicación se les mostró un fragmento del programa de televisión Redes, de Televisión Española (TVE), donde se explicaba la necesidad de poner en forma el cerebro (https://www.youtube.com/watch?v=vw5L-YNMewk). La segunda parte consistió en explicar que a medida que aprendemos se establecen más conexiones entre las neuronas, y que es común en los cerebros de científicos como Albert Einstein o Marie Curie que haya una red densa de neuronas conectadas. También en esta ocasión, la explicación se ilustró con un vídeo de TVE (https://www.youtube.com/watch?v=JR4gdLiuzQs) donde se explicaba de un modo muy gráfico cómo al aprender cosas nuevas se generan conexiones entre las neuronas. En la tercera parte de la sesión se habló del cociente intelectual, y cómo este concepto ha dejado de tener validez para muchos profesionales e investigadores porque se han encontrado evidencias de que la inteligencia no es estable, sino que se puede modificar y, por tanto, el resultado de nuestro cociente intelectual, al no tratarse de una capacidad inmutable, se puede modificar. A continuación, se proyectó un fragmento del programa Redes donde el presentador entrevistaba a un experto en el campo, Shlomo Breznitz, sobre este tema (https://www.youtube.com/watch?v=jUeix7OWP3Q). Por último, el objetivo de la cuarta parte de la sesión fue consolidar lo aprendido. Los alumnos debían redactar una carta en una situación hipotética: tenían que imaginarse que años después, estudiando bachillerato, un amigo o su pareja les contaba que quería dejar de estudiar porque no se sentía suficientemente inteligente como para afrontar los estudios, y ellos tenían que convencerle de que no tomara esa decisión aludiendo a lo aprendido durante la sesión.

\section{Análisis de datos}

\section{Análisis preliminar}

En primer lugar, para cada uno de los alumnos se calculó la media de los tres ítems en cada una de las tres evaluaciones o momentos. A continuación, para facilitar la interpretación, se calculó la puntuación diferencial, es decir, de la puntuación media de cada sujeto en los tres momentos, se restó la gran media, que es la media calculada teniendo en cuenta todas las 
puntuaciones de toda la muestra en las tres evaluaciones. Se calculó la puntuación media y la desviación típica del grupo experimental y control en cada una de las tres evaluaciones. Y se realizaron dos contrastes unilaterales a través de una prueba T-Student para muestras relacionadas con los sujetos del grupo experimental: el primero, para analizar si después de la intervención, la disminución de la variable dependiente había sido significativamente diferente de cero, y el segundo, para analizar si la variable dependiente volvía a subir en la tercera evaluación. Asimismo, se realizaron dos contrastes de hipótesis bilaterales, también a través de pruebas T-Student para muestras relacionadas con los sujetos del grupo control, con el fin de analizar si había diferencias significativas entre la primera y la segunda evaluación, y entre ésta última y la tercera. Por último, como indicador del tamaño del efecto, se calculó la d de Cohen en cada comparación de medias.

\section{Análisis multinivel}

Seguidamente, para analizar si la intervención había sido eficaz se realizó un análisis multinivel, que, a diferencia de un análisis de varianza de medidas repetidas, ofrece cuatro ventajas: 1) el tiempo entre las evaluaciones no tiene que ser el mismo, 2) no hay que eliminar los sujetos con datos perdidos en alguna de las evaluaciones, 3) no exige igualdad en las varianzas de cada evaluación, 4) tampoco se precisa igualdad en la covarianza entre las evaluaciones (Kwok et al., 2008).

Para analizar qué parte de la variabilidad total de las tres evaluaciones se debía a diferencias entre los participantes, se calculó el coeficiente de correlación intraclase (ICC, por sus siglas en inglés), que mide la relación entre variabilidad de las puntuaciones individuales de cada sujeto y la variabilidad total de todos los sujetos del estudio. Para obtener evidencias de que la intervención ha sido eficaz o no hay que demostrar que los sujetos de ambos grupos siguen trayectorias diferentes. En este caso, los datos de los sujetos seguían trayectorias curvilíneas, por lo que se construyó un modelo multinivel de crecimiento cuadrático con el grupo (control y experimental) como covariable, con la siguiente función: $Y_{\mathrm{ti}}=\gamma_{0 \mathrm{i}}+$ $\gamma_{1 i} \cdot$ tiempo $+\gamma_{2 i} \cdot$ tiempo $^{2}+\gamma_{3 i} \cdot$ grupo $+\gamma_{4 i} \cdot$ tiempo $\cdot$ grupo $+\gamma_{5 i} \cdot$ tiempo $^{2} \cdot$ grupo. Al ser pequeño el tamaño de la muestra, como método de estimación se utilizó el de máxima verosimilitud restringida (Kwok et al., 2008).

Por último, para analizar en qué medida la intervención fue eficaz (tamaño del efecto) se calculó la Pseudo R cuadrada, que mide la proporción de varianza explicada por la 
covariable, es decir, mide qué porcentaje de la variabilidad de las trayectorias de cada sujeto se debe a la intervención. Para calcular la Pseudo R cuadrada se compara la varianza entre sujetos del modelo con covariable, con la varianza entre sujetos del modelo sin tener en cuenta la covariable grupo. Los análisis de datos fueron realizados con el programa informático SPSS 22.

\section{Resultados}

\section{Análisis preliminar}

En la Tabla 1 se puede observar la media de las puntuaciones diferenciales de los dos grupos en las tres evaluaciones. Prestando atención a los datos del grupo experimental, si comparamos la primera con la segunda evaluación, se puede ver que la media de la percepción de la inteligencia como algo fijo disminuye tras la realización de la intervención, $[\mathrm{t}(22)=-1.66, p=.05, \mathrm{~d}=.32]$ pero vuelve a aumentar en la tercera evaluación [ $t(23)=-$ $1.79, p=.04, \mathrm{~d}=.39]$. Sin embargo, en el grupo control no se observan diferencias significativas entre la primera y la segunda evaluación $[t(20)=-.88, p=.19, d=.18$, ni entre la segunda y la tercera evaluación $\mathrm{t}(20)=-1.07, p=.15, d=.13$ ]

Tabla 1

Puntuación media y desviación típica por grupo y evaluación

\begin{tabular}{cccccccc}
\hline Grupo & \multicolumn{7}{c}{ Evaluación } \\
& \multicolumn{2}{c}{1} & \multicolumn{2}{c}{2} & \multicolumn{2}{c}{3} \\
& M & DT & M & DT & M & DT \\
Experimental & .04 & 1.53 & -.44 & 1.51 & .04 & 1.57 \\
Control & .02 & 1.70 & .32 & 1.61 & .11 & 1.59 \\
\hline
\end{tabular}

\section{Análisis multinivel}

El ICC obtuvo un valor de .50 , es decir, el $50 \%$ de la variación total a lo largo de las tres evaluaciones fue debida a diferencias entre los participantes y el otro $50 \%$ debido a diferencias intrapersonales. En la Tabla 2 se pueden ver los parámetros de la función y su nivel de significación. Sustituyendo los parámetros en la función descrita en el apartado de análisis de datos, obtenemos: $\mathrm{Y}_{\mathrm{ti}}=4.29+(-5.54) \cdot$ tiempo $+1.36 \cdot$ tiempo $^{2}+(-2.55) \cdot$ grupo + $3.30 \cdot$ tiempo $^{\text {grupo }}+\left(-0.81 \cdot\right.$ tiempo $^{2} \cdot$ grupo $)$. 
Tabla 2

Parámetros del modelo multinivel

\begin{tabular}{|c|c|c|c|}
\hline & Parámetro & Error típico & $p$ \\
\hline \multicolumn{4}{|l|}{ Efectos fijos } \\
\hline Intercepto $\left(\gamma_{0 i}\right)$ & 4.29 & 2.07 & .00 \\
\hline Tiempo $\left(\gamma_{1 i}\right)$ & -5.54 & 2.24 & .02 \\
\hline $\operatorname{Tiempo}^{2}\left(\gamma_{2 \mathrm{i}}\right)$ & 1.36 & .55 & .02 \\
\hline Grupo $\left(\gamma_{3 i}\right)$ & -2.55 & 1.34 & .06 \\
\hline Tiempo·Grupo $\left(\gamma_{4 \mathrm{i}}\right)$ & 3.30 & 1.44 & .03 \\
\hline Tiempo $^{2} \cdot$ Grupo $\left(\gamma_{5 i}\right)$ & -.81 & .35 & .03 \\
\hline \multicolumn{4}{|l|}{ Efectos aleatorios } \\
\hline$\sigma^{2}$ intra sujetos $\left(\tau_{00}\right)$ & 0.93 & & \\
\hline$\sigma^{2}$ entre sujetos $\left(\sigma^{2}\right)$ & 2.34 & & \\
\hline
\end{tabular}

A través del análisis multinivel se observó que la trayectoria del grupo experimental era diferente a la del grupo control, ya que la interacción entre la variable tiempo al cuadrado $\mathrm{y}$ grupo fue significativa, $p=.03$, y el tamaño del efecto, calculado a través de la Pseudo $\mathrm{R}$ cuadrada, fue .09, lo que implica que la intervención tuvo un efecto medio. En la Figura 1 se representan las trayectorias de cada grupo a través de las puntuaciones estimadas por medio de la función previamente descrita. Se puede observar cómo en el grupo experimental disminuye la puntuación de la variable dependiente después de la intervención, pero vuelve a recuperarse con el paso del tiempo. Sin embargo, la puntuación del grupo control no disminuyó entre la primera y la segunda evaluación y, aunque a simple vista puede parecer que ha aumentado, esta diferencia, como se describió en la sección de análisis preliminares, no fue significativa $[\mathrm{t}(20)=-.88, p=.19]$. 


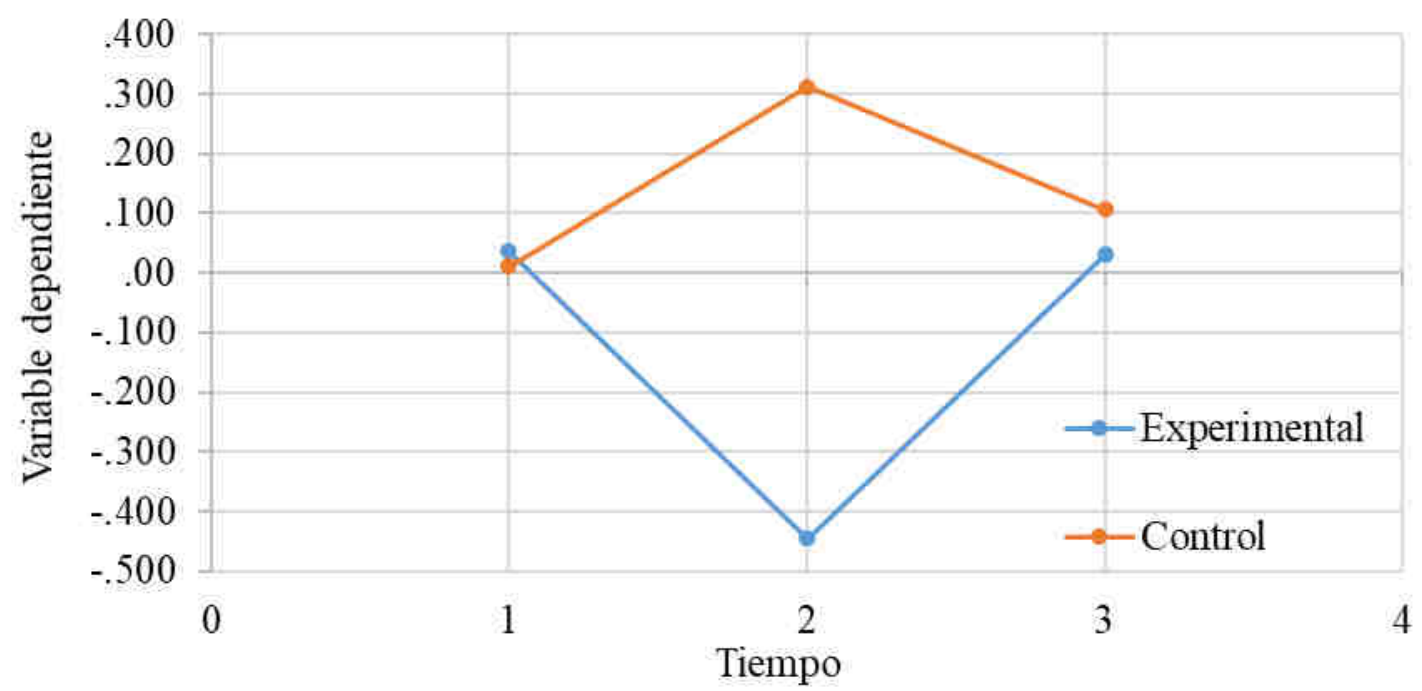

Figura 1. Puntaciones medias estimadas por grupo y tiempo

\section{Discusión}

El objetivo de este trabajo fue analizar la eficacia de una intervención para que el alumnado de Educación Secundaria pensara en la inteligencia como algo que se puede modificar. Hipotetizamos que el grupo experimental modificaría su percepción sobre su inteligencia tras la intervención. Nuestra hipótesis se cumplió de forma parcial, ya que, aunque los resultados tras la intervención mostraron que el objetivo se había conseguido, es decir, el grupo experimental cambió su idea sobre la inteligencia, pensando en ella como una capacidad modificable, sin embargo, un mes después de la intervención el grupo volvió a sus ideas iniciales.

Los resultados de este trabajo están en línea con los obtenidos por Donohoe et al. (2012), quienes observaron que, aunque la intervención había sido eficaz, con el paso del tiempo los efectos de la intervención fueron desvaneciéndose, ya que los alumnos volvieron a pensar en la inteligencia como antes de la intervención. Si tenemos en cuenta los resultados de la intervención realizada por Blackwell et al. (2007), habría que destacar que la variable dependiente en ese estudio (las calificaciones de la asignatura de Matemáticas) fue evaluada de forma diferente, ya que se tomaron dos medidas previas y solo una posterior a la intervención, por lo que surge la pregunta sobre si pasado el tiempo el efecto de la intervención se habría desvanecido. A raíz de los resultados de nuestro estudio y de los obtenidos por Donohoe et al. (2012), parece obvio que en este tipo de intervenciones breves 
es necesario dedicar algo de tiempo a consolidar lo trabajado con los alumnos. Parece que estas intervenciones breves pueden no ser eficaces de forma continuada en el tiempo, sino en momentos puntuales, por ejemplo, en época de exámenes, ya que en estas fechas aumentan los sentimientos de incompetencia, sobre todo en los alumnos con bajo rendimiento. También es destacable el aumento de la percepción de la inteligencia como algo fijo en el grupo control. Otros estudios longitudinales que han analizado mindsets u orientaciones de metas no han observado una variabilidad tan alta (Martin, 2015; Martin y Liem, 2010), además el período entre evaluacioens de estos estudios ha sido mayor que el nuestro. Por lo tanto, puede que esta variablidad, que no fue significativa, se debe simplemente al azar, aun así, consideramos que sería interesante realizar estudios longitudinales para conocer la trayectoria de los mindsets.

En este trabajo queremos nombrar algunas limitaciones y proponer ideas para futuros estudios. En primer lugar, aunque se ha contado con un diseño con grupo cuasi-experimental y grupo cuasi-control, lo que aumenta la validez del estudio en comparación con un diseño pre-post de un solo grupo, debemos señalar que, al igual que sucede en otros estudios similares (Donohoe et al., 2012; Blackwell et al., 2007; McGinley y Jones, 2014), el número de sujetos por grupo era reducido, por lo que hay que ser cautos a la hora de hacer inferencias. En segundo lugar, futuras investigaciones podrían probar el efecto de refuerzos, semanales, bisemanales o mensuales, y analizar si el cambio en la percepción sobre la inteligencia de los alumnos se mantiene o vuelve a su estado original.

En tercer lugar, como perspectiva de futuro, creemos que sería interesante analizar si la eficacia de esta intervención sería mayor si se aplica en alumnos que se sienten inferiores o menos capaces que sus compañeros; esta idea la plantean Davis, Burnette, Allison y Stone (2010), quienes observaron que el pensar en la inteligencia como algo maleable era clave cuando los estudiantes, en una competición matemática, creían que eran menos capaces que el resto de sus compañeros. También Chen y Pajares (2010) señalan que pensar en la inteligencia como algo que se puede cambiar es clave cuando los alumnos empiezan a bajar su rendimiento, por lo que futuras investigaciones podrían analizar si esta intervención sería más eficaz en alumnos que han visto disminuido su rendimiento académico o con alumnos inmigrantes ya que el riesgo de fracaso escolar es mayor (Sandín-Esteban y Sánchez-Martí, 2015). En cuarto lugar, hay que resaltar que en este estudio utilizamos una selección de ítems del instrumento para evaluar la percepción sobre la inteligencia, aunque aportamos evidencias 
de fiabilidad y, consideramos que los ítems representan de forma adecuada el constructo a evaluar, podría darse que los resultados con otros ítems fueran algo diferentes.

Por último, queremos subrayar que con este trabajo hemos contribuido a aumentar el conocimiento de las intervenciones breves en el contexto educativo (Yeager et al., 2013; Yeager y Walton, 2011), útiles para disminuir la desmotivación académica, problema muy importante en el actual sistema educativo (González y Paoloni, 2015; OECD, 2012). En este trabajo se diseñó una actividad breve que modificó un componente motivacional con una fuerte relación con el funcionamiento académico (Blackwell et al., 2007) y lo destacable es que puede servir como punto de partida para que futuras investigaciones mejoren el rendimiento académico de una forma sencilla, replicable y fácilmente escalable. 


\section{Referencias}

Anderman, L. H. (2003). Academic and social perceptions as predictors of change in middle school students' sense of school belonging. The Journal of Experimental Education, 72(1), 5-22. doi:10.1080/00220970309600877

Aronson, J., Fried, C. B. y Good, C. (2002). Reducing the effects of stereotype threat on African American college students by shaping theories of intelligence. Journal of Experimental Social Psychology, 38(2), 113-125. doi:10.1006/jesp.2001.1491

Blackwell, L. S., Trzesniewski, K. H. y Dweck, C. S. (2007). Implicit theories of intelligence predict achievement across an adolescent transition: A longitudinal study and an intervention. Child Development, 78(1), 246-63. doi:10.1111/j.1467-8624.2007.00995.x

Chen, J. A. y Pajares, F. (2010). Implicit theories of ability of Grade 6 science students: Relation to epistemological beliefs and academic motivation and achievement in science. $\begin{array}{llll}\text { Contemporary } \quad \text { Educational } & \text { 75-87. }\end{array}$ doi:10.1016/j.cedpsych.2009.10.003

Cohen, G. L., Garcia, J., Purdie-Vaughns, V., Apfel, N. y Brzustoski, P. (2009). Recursive processes in self-affirmation: Intervention to close the minority achievement gap. Science, 324, 400-403.

Cordero, J. M. y Manchón, C. (2014). Explanatory factors for achievement in primary education: An analysis using timss 2011. Estudios Sobre Educación, 27, 9-35. doi:10.15581/004.27.9-35

Crane, C. A. y Eckhardt, C. I. (2013). Evaluation of a single-session brief motivational enhancement intervention for partner abusive men. Journal of Counseling Psychology, 60(2), 180-7. doi:10.1037/a0032178

Davidson, A. J., Gest, S. D. y Welsh, J. A. (2010). Relatedness with teachers and peers during early adolescence: An integrated variable-oriented and person-oriented approach. Journal of School Psychology, 48(6), 483-510. doi:10.1016/j.jsp.2010.08.002

Davis, J. L., Burnette, J. L., Allison, S. T. y Stone, H. (2010). Against the odds: Academic underdogs benefit from incremental theories. Social Psychology of Education, 14(3), 331-346. doi:10.1007/s11218-010-9147-6

Donohoe, C., Topping, K. y Hannah, E. (2012). The impact of an online intervention (Brainology) on the mindset and resiliency of secondary school pupils: a preliminary mixed methods study. Educational Psychology, 32(5), 641-655. doi:10.1080/01443410.2012.675646 
Dweck, C. S. (1999). Self-theories: Their role in motivation, personality, and development. Philadelphia: Psychology Press.

Dweck, C. S. (2006). Mindset: The new psychology of success. New York: Random House.

Dweck, C. S. y Leggett, E. (1988). A social-cognitive approach to motivation and personality. Psychological Review, 95(2), 256-273. Retrieved from http://psycnet.apa.org/journals/rev/95/2/256/

Eccles, J. S. (1983). Expectancies, values, and academic behaviours. En J. T. Spence (Ed.), Achievement and achievement motives (pp. 75-146). San Diego, CA: Freeman.

Erdley, C. A. y Dweck, C. S. (1993). Children's implicit personality theories as predictors of their social judgments. Child Development, 64(3), 863-78.

Erdley, C. A., Loomis, C. C., Cain, K. M., Dumas-Hines, F. y Dweck, C. S. (1997). Relations among children's social goals, implicit personality theories, and responses to social failure. Developmental Psychology, 33(2), 263-272. doi:10.1037//0012-1649.33.2.263

González, A. y Paoloni, P. (2015). Implicación y rendimiento en Física: el papel de las estrategias docentes en el aula , y el interés personal y situacional del alumnado. Revista de Psicodidáctica, 20(1), 25-45. doi:10.1387/RevPsicodidact.11370

Goodenow, C. (1993). Classroom belonging among early adolescent students: Relationships to motivation and achievement. The Journal of Early Adolescence, 13(1), 21-43.

Hong, Y., Chiu, C., Dweck, C. S., Lin, D. M.-S. y Wan, W. (1999). Implicit theories, attributions, and coping: A meaning system approach. Journal of Personality and Social Psychology, 77(3), 588-599. doi:10.1037//0022-3514.77.3.588

Hulleman, C. S. y Harackiewicz, J. M. (2009). Promoting interest and performance in high school science classes. Science, 326(5958), 1410-1412. doi:10.1126/science.1177067

Inglés, C. J., Martínez-Monteagudo, M. C., García-Fernández, J. M., Valle, A., Núñez, J. C., Delgado, B. y Torregrosa, S. (2015). Motivational profiles Spanish students of compulsory secondary education: differential analysis of academic self-attributions. Anales de Psicología, 31(2), 579-588.

Kwok, O.-M., Underhill, A. T., Berry, J. W., Luo, W., Elliot, T. R. y Yoon, M. (2008). Analyzing longitudinal data with multilevel models: An example with individuals living with lower extremity intra-articular fractures. Rehabilitation Psychology, 53(3), 370386. doi:10.1037/a0012765

Lazowski, R. A., \& Hulleman, C. S. (2016). Motivation interventions in education: A metaanalytic review. Review of Educational Research, (En prensa). doi:10.3102/0034654315617832 
Lim, M., Tormshak, E. a. S. y Dishion, T. J. (2005). A one-session intervention for parents of young adolescents: Videotape modeling and motivational group discussion. Journal of Emotional and Behavioral Disorders, 13(4), 194-199. doi:10.1177/10634266050130040101

Locke, E. A. y Latham, G. P. (1990). A theory of goal setting and task performance. Englewood Cliffs, NJ: Prentice Hall.

Locke, E. A. y Latham, G. P. (2002). Building a practically useful theory of goal setting and task motivation: A 35-year odyssey. American Psychologist, 57(9), 705-717. doi:10.1037//0003-066X.57.9.705

Luo, W., Lee, K., Ng, P. T. y Wei Ong, J. X. (2014). Incremental beliefs of ability, achievement emotions and learning of Singapore students. Educational Psychology, 34(5), 619-634. doi:10.1080/01443410.2014.909008

Martin, A. J. (2015). Implicit theories about intelligence and growth (personal best) goals: Exploring reciprocal relationships. British Journal of Educational Psychology, 85(2), 207-223. doi:10.1111/bjep.12038

Martin, A. J. y Liem, G. A. D. (2010). Academic personal bests (PBs), engagement, and achievement: A cross-lagged panel analysis. Learning and Individual Differences, 20(3), 265-270. doi:10.1016/j.lindif.2010.01.001

McGinley, J. J. y Jones, B. D. (2014). A brief instructional intervention to increase students' motivation on the first day of class. Teaching of Psychology, 41(2), 158-162. doi:10.1177/0098628314530350

Morisano, D., Hirsh, J. B., Peterson, J. B., Pihl, R. O. y Shore, B. M. (2010). Setting, elaborating, and reflecting on personal goals improves academic performance. The Journal of Applied Psychology, 95(2), 255-64. doi:10.1037/a0018478

OCDE. (2012). Orientaciones de PISA para las Islas Canarias, España: Sistemas fuertes y reformadores exitosos en la educación. OECD Publishing. doi:10.1787/9789264174788es

Paunesku, D., Walton, G. M., Romero, C., Smith, E. N., Yeager, D. S. y Dweck, C. S. (2015). Mind-set interventions are a scalable treatment for academic underachievement. Psychological Science, 26(6), 784-793. doi: 10.1177/0956797615571017

Rattan, A., Good, C. y Dweck, C. S. (2012). "It's ok - Not everyone can be good at math": Instructors with an entity theory comfort (and demotivate) students. Journal of Experimental Social Psychology, 48(3), 731-737. doi:10.1016/j.jesp.2011.12.012 
Roeser, R. W. y Eccles, J. S. (1998). Adolescents' perceptions of middle school: relation to longitudinal changes in academic and psychological adjustment. Journal of Research on Adolescence, 8(1), 123-158. doi:10.1207/s15327795jra0801_6

Romero, C., Master, A., Paunesku, D., Dweck, C. S. y Gross, J. J. (2014). Academic and emotional functioning in middle school: The role of implicit theories. Emotion, 14(2), 227-234. doi:10.1037/a0035490

Sandín-Esteban, M. P. y Sánchez-Martí, A. (2015). Resilience and school success of young immigrants. Infancia y Aprendizaje: Journal for the Study of Education and Development, 38(1), 209-211. doi:10.1080/02103702.2015.1009232

Spitzer, B. y Aronson, J. (2015). Minding and mending the gap: Social psychological interventions to reduce educational disparities. British Journal of Educational Psychology, 85, 1-18. doi:10.1111/bjep.12067

Thurstone, C., Riggs, P. D., Klein, C. y Mikulich-Gilbertson, S. K. (2007). A one-session human immunodeficiency virus risk-reduction intervention in adolescents with psychiatric and substance use disorders. Journal of the American Academy of Child \& Adolescent Psychiatry, 46(9), 1179. doi:10.1097/chi.0b013e31809fe774

Verniers, C. y Martinot, D. (2015). Perception of students' intelligence malleability and potential for future success: Unfavourable beliefs towards girls. British Journal of Educational Psychology, in press. doi:10.1111/bjep.12073

Walton, G. M. y Cohen, G. L. (2011). A brief social-belonging intervention improves academic and health outcomes of minority students. Science, 331, 1447-1451.

Wentzel, K. R. (2009). Peers and academic functioning at school. En K. H. Rubin, W. M. Bukowski y B. Laursen (Eds.), Handbook of peer interactions, relationships, and groups (pp. 531-547). New York, US: The Guilford Press.

Wigfield, A. y Eccles, J. S. (2000). Expectancy-value theory of achievement motivation. Contemporary Educational Psychology, 25(1), 68-81. doi:10.1006/ceps.1999.1015

Yeager, D. S., Johnson, R., Spitzer, B. J., Trzesniewski, K. H., Powers, J. y Dweck, C. S. (2014). The far-reaching effects of believing people can change: implicit theories of personality shape stress, health, and achievement during adolescence. Journal of Personality and Social Psychology, 106(6), 867-884. doi:10.1037/a0036335

Yeager, D. S., Paunesku, C., Dave, R., Hulleman, C. S., Schneider, B., Hinojosa, C., ... Dweck, C. S. (2016). Designing social-psychological interventions for full-scale implementation: The case of the growth mindset during the transition to high school. Journal of Educational Psychology, 108(3), 374-391. doi: 10.1007/s13398-014-0173-7.2 $-396-$ 
Yeager, D. S. y Walton, G. M. (2011). Social-Psychological interventions in education: They're not magic. Review of Educational Research, 81(2), 267-301. doi:10.3102/0034654311405999

Yeager, D. S., Walton, G. M. y Cohen, G. L. (2013). Addressing achievement gaps with psychological interventions. Phi Delta Kappan, 94(5), 62-65. 